\title{
BIOACTIVITE DES EXTRAITS FOLIAIRES DE RUTA CHALEPENSIS L. (RUTACEAE) SUR LA MORTALITE DES LARVES DE CULISETA LONGIAREOLATA (DIPTERA, CULICIDAE)
}

\author{
S. Benhissen*1,2, W. Habbachi ${ }^{2}$, K. Rebbas ${ }^{1,3}$, F. Masna ${ }^{4}$ \\ ${ }^{1}$ Département des sciences de la nature et de la vie, faculté des sciences, université \\ Mohamed Boudiaf de M'Sila, 28 000, Algérie. \\ ${ }^{2}$ Laboratoire Neuroendocrinologie Appliquée. Département de Biologie, Faculté des \\ Sciences, BP 12 Université Badji Mokhtar - 23000 Annaba, Algérie. \\ ${ }^{3}$ Laboratoire d'Agro-Biotechnologie et de nutrition en zones arides et semi arides/ \\ Equipe de recherche de gestion des ressources naturelles et environnement. \\ Université Ibn Khaldoun, Tiaret, Algérie. \\ ${ }^{4}$ Laboratoire de Désertification et climat, Université Amar Telidji, 03000 Laghouat, \\ Algérie. \\ *Correspanding author: Benhissen Saliha
}

(Received August 2017 - Accepted January 2019)

\section{RESUME}

S. Benhissen, W. Habbachi, K. Rebbas, F. Masna. 2019. Bioactivité des extraits foliaires de Ruta Chalepensis L. (Rutaceae) sur la mortalité des larves de Culiseta Longiareolata (Diptera, Culicidae). Journal Scientifique Libanais. 20(1): 1-9.

L'effet de l'extrait aqueux de Ruta chalepensis L.., plante spontanée du littoral algérien à des concentrations $(8 \mathrm{~g} / \mathrm{l}, 18,44 \mathrm{~g} / \mathrm{l}$ et $33,2 \mathrm{~g} / \mathrm{l})$, a été observé sur les larves de quatrième stade du Culiseta longiareolata (Macquart) sous des conditions contrôlées. Les résultats montrent que le taux de mortalité augmente en fonction de la concentration de l'extrait et du temps. Le taux de mortalité le plus important (100\%) a été obtenu après 3 jours de traitement pour la concentration la plus élevée, avec des concentrations létales CL50\% et CL90\% sont 8,82 g/l et 14,31 g/l, respectivement. Alors que le temps létal, le minimum (0,66 jours) a été enregistré avec la dose 33,2g/l. Certains composés de Ruta chalepensis pourraient être utiles dans l'élaboration ou la synthèse de bio-insecticides, dans le cadre d'une lutte intégrée contre les moustiques.

http://dx.doi.org/10.22453/LSJ-020.1.001-009

National Council for Scientific Research - Lebanon 2018@

1sj.cnrs.edu.lb/vol-20-no-1-2019/ 
Mots-clés: Culiseta longiareolata, Ruta chalepensis, extrait aqueux, insecticides.

\begin{abstract}
S. Benhissen, W. Habbachi, K. Rebbas, F. Masna. 2019. Bioactivity of foliar extracts of Ruta Chalepensis L. (Rutaceae) on larval mortality of Culiseta Longiareolata (Diptera, Culicidae). Lebanese Science Journal. 20(1): 1-9.
\end{abstract}

The effects of the aqueous extract of Ruta chalepensis L., a spontaneous plant on the Algerian coastline, used at concentrations of $8 \mathrm{~g} / \mathrm{l}, 18.44 \mathrm{~g} / \mathrm{l}$ and $33.2 \mathrm{~g} / \mathrm{l}$, were observed on the fourth instars larvae of Culiseta longiareolata (Macquart) under controlled conditions. The results showed that mortality rate increased with concentration of the extract and time. The highest mortality rate (100\%) was obtained after 3 treatment days in using the highest concentration; lethal concentrations LC50\% and $L C 90 \%$ are $8,82 \mathrm{~g} / \mathrm{l}$ and $14,31 \mathrm{~g} / \mathrm{l}$, respectively. So, the shortest lethal time $(0,66$ days) was recorded with the dose of $33.2 \mathrm{~g} / \mathrm{l}$. Hence, some compounds of Ruta chalepensis may be useful in the development or synthesis of bio-insecticides as a part of integrated fight against mosquitoes.

Keywords: Culiseta longiareolata, Ruta chalepensis, water extract, insecticides.

\title{
INTRODUCTION
}

Depuis l'antiquité, l'homme a cherché des moyens de protection contre les piqûres d'insectes vectorielles. Les premières stratégies de lutte intégrée sont essentiellement basées sur des matières actives des insecticides appartenant aux familles suivantes : les organophosphorés, les pyrethrinoides de synthèse, les organochlorés et les carbamates de synthèse. Ces préparations, bien qu'elles se soient révèles très efficaces sur les moustiques, présentent plusieurs inconvénients. L'accumulation significative de matières actives dans les écosystèmes aquatiques et terrestres traités présente un problème de pollution (Barbouche et al., 2001).

Pour assurer une meilleure intervention, tout en préservant au maximum le milieu naturel, de nouvelles méthodes préventives ainsi que de nouveaux produits sont constamment recherchés. Les substances naturelles qui présentent un large spectre d'action en pharmacologie comme anti-paludisme, bactéricides, fongicides, acaricides, etc., peuvent être aussi utilisées comme insecticides alternatifs.

L'utilisation des extraits de plantes comme insecticides, est connue depuis longtemps. En effet, le pyrèthre, la nicotine et la roténone sont déjà connus comme agents de lutte contre les insectes (Crosby et al., 1966). 
En Algérie, les études menées sur l'activité insecticides des extraits végétaux vis-avis des larves de moustiques sont très limitées. En effet, l'exception des travaux de Habbachi et al. (2014) et de Benhissen et al. (2015), Merabti et al (2015), peu d'étude ont été effectuée sur l'effet des extraits de plantes du Mali sur les larves de moustiques.

Les espèces de Rutaceae ont, aussi, attiré beaucoup d'attention en raison de leurs activités biologiques induites par les métabolites secondaires, et leurs propriétés antifongiques, antioxydantes et anti-inflammatoires (Gonzalez-Trujano et al., 2006 ; Iauk et al., 2004 ; Kabouche et al., 2003 ; Meepagala et al., 2005 ; Milesi et al., 2001 ; Raghav et al., 2006). Ruta chalepensis est une espèce très abondante dans la région méditerranéenne, communément appelée rue frangée (Iauk et al., 2004). Cette plante vivace est caractérisée par des feuilles ovales, grandes, pennées et bleu-vert qui ont de nombreux lobes oblongs lancéolés. L'inflorescence de cette espèce est sous la forme de cyme (Ben Bnina et al., 2010).

Cette plante est potentiellement toxique et carcinogène lorsqu' elle est administrée par voie orale, et peut provoquer une dermatite de contact. Les principaux composants qui ont été isolés de $R$. chalepensis sont des alcaloïdes (racine) ainsi que la chalépensine (parties aériennes) (Waterman, 1975). Les plantes servent à éloigner les chiens et les chats car ils en détestent l'odeur. De même, les feuilles séchées et écrasées constituent un insectifuge efficace (Attou, 2011).

Dans cet axe, notre travail a été réalisé pour apporter notre contribution pour une meilleure connaissance de la sensibilité des larves du Culiseta longaireolata aux extraits de la plante Ruta chalepensis.

\section{MATERIEL ET METHODES}

\section{Echantillonnage et élevage des moustiques}

Les larves de Culiseta longiareolata ont été collectées dans les différentes gites de la région de Boussaâda (Oued Boussaâda, M'sila - Algérie) (35'13'09" nord, $4^{\circ} 10^{\prime} 54^{\prime \prime}$ est), puis emmenées au laboratoire pour l'élevage de masse. L'élevage des larves récoltées a été maintenu au laboratoire dans des récipients contenants $250 \mathrm{ml}$ d'eau de source à $27^{\circ} \mathrm{C}, 70$ à $85 \%$ d'humidité avec une scotophase de 12:12. Les larves ont été nourris d'un mélange de biscuit et levure séchée ; tandis que les adultes ont été nourris de raisins secs. 


\section{Matériel végétal}

Ruta chalepensis a été récolté au stade floraison dans la région de Béjaia (Algérie) pendant le printemps. La plante récoltée est emmenée au laboratoire pour extraction des feuilles fraiches.

\section{Préparation des extraits végétaux}

D'après la méthode d'Alaoui Boukhris, (2009) on a préparé l'extrait aqueux de la plante à partir du mélange $100 \mathrm{~g}$ de feuilles fraiches de $R$. chalepensis préalablement séchées et coupées en menus morceaux, dans un 1 litre d'eau distillée et on laisse bouillir pendant une $1 \mathrm{~h}$ et 15 minutes sur une plaque chauffante à $180^{\circ} \mathrm{C}$. et $\left.a l, 2004\right)$. Le mélange obtenu est filtré à l'aide du papier filtre et on récupère une solution avec la concentration $166.67 \mathrm{~g} / \mathrm{l}$.

\section{Traitement}

Le traitement de $C$. longiareolata est inspiré de la technique des tests de sensibilité normalisée par l'Organisation Mondiale de la Santé (OMS, 1963). Les tests sont réalisés dans des béchers d'une capacité de $250 \mathrm{ml}$ contenant chacun 20 larves du quatrième stade de $C$. longiareolata (L4) dans $200 \mathrm{ml}$ d'eau de source. Après un test préliminaire on a administré trois concentrations aux larves du stade $\mathrm{L} 4(8 \mathrm{~g} / \mathrm{l}, 18,44 \mathrm{~g} / \mathrm{l}$ et $33,2 \mathrm{~g} / \mathrm{l})$. Ces valeurs sont choisies dans le but de chercher les doses létales. Chaque concentration est appliquée sur 3 répétitions, avec une préparation de 20 larves de $C$. longiareolata comme témoin.

La variable mesurée quotidiennement, pendant 15 jours, est le nombre d'individus morts (larves de L4, nymphes et adultes).

Les concentrations létales et les temps létaux (CL50\%, CL90\%, TL50\% et TL90\%) ont été calculés selon les procédés mathématiques de Finney (1971). Les données sont transformées et normalisées d'après les tables de Bliss; les calculs sont réalisés sur XLStat 2009.

\section{RESULTATS ET DISCUSSION}

\section{Observation générale}

L'observation des larves à l' œil nu montre qu'elles sont mortes, mais lors de l'observation sous microscope on remarque que les larves respirent et elles arrivent à survivre pendant quelques jours. 
Effet du $R$. chalepensis sur la mortalité des larves

La sensibilité des larves du quatrième stade de $C$. longiareolata aux extraits aqueux des feuilles de $R$. chalepensis se traduit par des taux de mortalité plus ou moins élevés selon les concentrations utilisées et surtout selon le temps d'exposition aux extraits (Tableau 1).

Le taux de mortalité varie entre $5 \%$ et $45 \%$ pour la concentration la plus faible $(8 \mathrm{~g} / \mathrm{l})$ alors qu'il arrive jusqu'à $100 \%$ après 2 jours du traitement lorsqu'on expose les larves aux fortes concentrations $(33.2 \mathrm{~g} / \mathrm{l})$.

Les larves du $4^{\text {ème }}$ stade de $C$. longiareolata exposées aux extraits de cette rutacée présentent des taux de mortalité moyens ne sont pas significativement différents au bout de 1 jour et 2 jours. Par contre, après $3 \mathrm{j}$ du traitement, les taux de mortalité sont significativement différents $\left(\mathrm{F}_{\mathrm{obs}}=8,880 ; p: 0,016\right)$.

Tableau 1. Taux de mortalité corrigée des larves du 4ème stade de $C$. longiareolata traitées avec les extraits aqueux des feuilles de $R$. chalepensis.

\begin{tabular}{|c|c|c|c|c|c|}
\hline Concentrations $\quad$ Temps & jour 1 & jour 2 & jour 3 & Fobs & $p$ \\
\hline $33.2 \mathrm{~g} / \mathrm{l}$ & $81.7 \%$ & 100.0 & 100.0 & 16.000 & $0.004 * *$ \\
\hline $18.44 \mathrm{~g} / \mathrm{l}$ & $26.7 \%$ & 76.7 & 95.0 & 1.405 & 0.316 \\
\hline $8 \mathrm{~g} / \mathrm{l}$ & $5.0 \%$ & 25.0 & 45.0 & 0.211 & 0.816 \\
\hline Fobs & 1,973 & 5,347 & 8,880 & & \\
\hline$p$ & 0,220 & 0,046 & $0,016 *$ & & \\
\hline
\end{tabular}

Selon la méthode de Finey 1971, la comparaison des variances pour ces taux de mortalité se fait après une transformation angulaire des taux de mortalité. Et quant aux moyennes et les écart-type des pourcentages transformés ci-dessous le tableau suivant qui résume ces données:

\begin{tabular}{|l|r|c|c|}
\hline $\begin{array}{r}\text { Temps } \\
\text { Concentrations }\end{array}$ & jour 1 & jour 2 & jour 3 \\
\hline $8 \mathrm{~g} / \mathrm{l}$ & $7,60 \pm 7,59$ & $28,49 \pm 7,78$ & $41,44 \pm 9,33$ \\
\hline $18 \mathrm{~g} / \mathrm{l}$ & $25,78 \pm 14,66$ & $63,10 \pm 9,43$ & $77,64 \pm 3,67$ \\
\hline $33,2 \mathrm{~g} / \mathrm{l}$ & $64,81 \pm 2,40$ & $84,26 \pm 0,00$ & $84,26 \pm 0,00$ \\
\hline
\end{tabular}




\section{Les paramètres toxicologiques}

Les résultats montrent qu'il y a une forte corrélation positive entre les taux de mortalité enregistrés et le temps d'exposition et/ou la concentration de l'extrait utilisée contre les moustiques (Tableau 2).

Pour assurer une mortalité de $50 \%$ des larves après 1 jour, la concentration de $R$. chalepensis doit être égale à $22,17 \mathrm{~g} / \mathrm{l}$, par contre $46 \mathrm{~g} / \mathrm{l}$ des feuilles assurent la mortalité de $90 \%$ (Tableau 2). Au deuxième jour, la CL50\% est de 11,16g/l, alors que la CL90\% est de 17,15g/l (Tableau 2) ; et après 3 jours de traitement, la CL50\% et la CL90\% ne dépassent pas les $8,82 \mathrm{~g} / 1$ et les $14,31 \mathrm{~g} / 1$, respectivement.

Concernant les temps létaux, la concentration $8 \mathrm{~g} / \mathrm{l}$ de feuilles de $R$. chalepensis peut éliminer $50 \%$ de la population de $C$. longiareolata en environ 3 jours et $90 \%$ de ces moustiques peuvent être éliminés dans 8 jours de traitement (Tableau 2). Lorsqu'on applique $18.44 \mathrm{~g} / 1$ d'extrait de $R$. chalepensis, le TL50\% est de 1,46 jour, alors que le TL90\% est de 3,58 jours. Concernant la concentration la plus élevée (33.2 g/l), les temps létaux calculés (TL50\% et TL90\%) sont aussi les plus courts puisqu'ils ne dépassent pas, respectivement 0,66 jour et les 1,05 jours.

Tableau 2. Paramètres toxicologiques des extraits aqueux des feuilles de $\boldsymbol{R}$. chalepensis.

\begin{tabular}{|c|c|c|c|}
\hline \multicolumn{4}{|c|}{ Temps d'exposition } \\
\hline Temps & 1 jour & 2 jours & 3 jours \\
\hline Droite de régression & $\begin{array}{l}Y=-0,41+4,02 X \\
R^{2}=0,995\end{array}$ & $\begin{array}{l}Y=-2,20+6,87 X \\
R^{2}=0,910\end{array}$ & $\begin{array}{l}Y=-0,77+6,10 X \\
R^{2}=0,980\end{array}$ \\
\hline CL50\% (g/l) & 22,17 & 11,16 & 8,82 \\
\hline CL90\% (g/l) & 46 & 17,15 & 14,31 \\
\hline \multicolumn{4}{|c|}{ Concentration utilisée } \\
\hline Concentration & $33,2 \mathrm{~g} / \mathrm{l}$ & $18,44 \mathrm{~g} / \mathrm{l}$ & $8 \mathrm{~g} / \mathrm{l}$ \\
\hline Droite de régression & $\begin{array}{l}Y=6,13+6,36 X \\
R^{2}=0,872\end{array}$ & $\begin{array}{l}Y=4.51+3.19 X \\
R^{2}=0,964\end{array}$ & $\begin{array}{l}Y=3,34+3.23 X \\
R^{2}=1,000\end{array}$ \\
\hline TL50\% & 0,66 & 1,42 & 3,26 \\
\hline TL90\% & 1,05 & $\mathbf{3 , 5 8}$ & 8,13 \\
\hline
\end{tabular}


Pour assurer une meilleure intervention, tout en préservant au maximum le milieu naturel, de nouvelles méthodes préventives ainsi que de nouveaux produits sont constamment recherchés.

Plusieurs travaux étudiant l'effet toxique des produits issus des végétaux contre les larves de moustiques. Nous citons à cet effet, les travaux d'Alouani et al. (2009) qui ont mis en évidence une activité larvicide de l'azadiractine (extrait d'arbre d'Azadirachta indica A. Juss.) sur les larves de $4{ }^{\text {ème }}$ stade de Culex pipiens (Linnaeus). Govindara et Bollipo (2013) ont testés avec succès l'activité larvicide des huiles essentielles d'Artemisia vulgaris L. (Asteraceae) sur Aedes aegypti (Linnaeus).

Dans notre recherche, la toxicité des extraits aqueux du $R$. chalepensis, a été étudiée sur les larves de $4{ }^{\text {éme }}$ stade de $C$. longiareolata. Nous avons utilisé trois doses à administrer $(33 \mathrm{~g} / \mathrm{l} ; 18 \mathrm{~g} / \mathrm{l} ; 8 \mathrm{~g} / \mathrm{l})$, chaque dose a été appliquée avec trois répétitions et un témoin. Pour cet extrait, nous avons analysé les effets de la concentration et du type d'extrait sur l'action larvicide.

Nos résultats montrent que $R$. chalepensis entraîne une mortalité variable selon la concentration utilisée et le temps de traitement. Nous avons démontré que les concentrations létales (CL50, CL90) diminuent en fonction de la durée du traitement. Au bout de 3 jours de traitement, les taux de mortalité des larves augmentent et peuvent atteindre $100 \%$ lorsqu'on utilise la plus forte concentration $33 \mathrm{~g} / 1$ et la CL50 est équivalente à $5,75 \mathrm{~g} / \mathrm{l}$, alors que CL90 est égale 11,48 g/l.

Beaucoup de travaux récents indiquent les effets insecticides des huiles essentiels extraits de Ruta chalepensis sur sur les larves du moustique Aedes albopictus Skuse (Conti et al., 2013), sur le Coléoptère Tribolium castaneum (Herbst) (Majdoub et al.,2014), , sur Culex pipiens (Abdel-Sattar et al. ,2015) et récemment sur Aegorhinus superciliosus (Guérin) (Coleoptera: Curculionidae) (Tampe et al., 2016).

Des études antérieures indiquent les effets des composés allélochimiques de $R$. chalepensis, capables de provoquer des perturbations physiologiques. C'est le cas des alcaloïdes qui dissuadent les insectes phytophages, affectent le système nerveux et la division cellulaire, et ont une propriété toxique et paralysante sur les insectes.

Des travaux similaires prouvent l'effet insecticide de $R$. chalepensis, un extrait à l'hexane des parties aériennes s'est révélé avoir une forte action molluscicide sur l'escargot vecteur de la schistosomose, Bulinus truncatus (Audouin) (Attou, 2011). 


\section{CONCLUSION}

La présente étude a montré que l'extrait aqueux de Ruta chalepensis possède des effets toxiques sur les larves de Culiseta longiareolata, surtout après traitement par la plus haute concentration $(33.2 \mathrm{~g} / \mathrm{l})$. Il est nécessaire de tester d'autres concentrations et d'autres méthodes d'extractions qui peuvent éventuellement donner de meilleurs résultats, ainsi que sur d'autres stades de développement de l'insecte. Cette rutacée présente une opportunité intéressante pour développer des bio-insecticides dans le cadre de programmes de lutte intégrée.

\section{RÉFÉRENCES}

Abdel-Sattar E., El Sayed A.M, Zaitoon A., Bakhashwain AA., 2015. Evaluation of Some Medicinal Plants for Control of Culex pipiens Mosquitoes. Research Journal of Pharmaceutical, Biological and Chemical Sciences. 6(1): 898-905.

Alaoui Boukhris M, 2009. Activités larvicides des extraits de plantes sur les larves de moustiques vecteurs de maladies parasitaires. Mémoire du master. Université Fès, Maroc.77p

Alouani, A., Rehimi, N. and Soltani, N. 2009. Larvicidal Activity of a Neem Tree Extract (Azadirachtin) Against Mosquito Larvae in the Republic of Algeria. Jord. J. Biol. Sci. 2(1): 15-22.

Attou, A. 2011. Contribution à l'étude phytochimique et activités biologiques des extraits de la plante Ruta chalepensis (Fidjel) de la région d'Ain Témouchent. Thèse de magister. Université Abou Bekr Belkaid Tlemcen, Algérie, 119 p.

Barbouche, N., Hajjem, B., Lognay, G. et Ammar, M. 2001. Contribution à l'étude de l'activité biologique d'extraits de feuilles de Cestrumparqui L'Hérit. (Solanaceae) sur le criquet pèlerin Schistoceracagregaria(Forsk.). Biotechnol. Agron. Soc. Environ. 5 (2): 85-90.

Ben Bnina, E., Hammami, S., Daami-Remadi, M., Ben Jannet, H., and Mighri, Z. 2010. Chemical composition and antimicrobial effects of Tunisian Ruta chalepensis $\mathrm{L}$. essential oils. Journal de la Société Chimique de Tunisie 12: 1-9.

Benhissen, S., Habbachi, W., Mecheri, H, Masna, F, Ouakid, ML. and Bairi, A.2015. Effects of Aqueous Extracts of Daphne gnidium (Thymelaeaceae) Leaves on Larval Mortality and Reproductive Performance of Adult Culex pipiens (Diptera; Culicidae). PhytoChem \& BioSub Journal, 9(2): 34-38.

Conti, B., Leonardi, M., Pistelli, L., Profeti, R., Ouerghemmi, I., Benelli, G. 2013. Larvicidal and repellent activity of essential oils from wild and cultivated Ruta chalepensis L. (Rutaceae) against Aedes albopictus Skuse (Diptera: Culicidae), an arbovirus vector. Parasitol. Res. 112: 991-999.

Crosbyd, G. 1966. Natural pest control Agents. Adv. Chem. Ser. 53: 1-16.

Finney D.J. 1971. Probits analysis. Ed. Cambridge University Press. London. 
Gonzalez-Trujano, M.E., Carrera, D., Ventura- Martinez, R., Cedillo-Portugal, E., Navarrete, A. 2006. Neuropharmacological profile of an ethanol extract of Ruta chalepensis L. in mice. J. Ethnopharm. 106: 129-135.

Govindaraj S et Bollipo D. R K. 2013. Composition and Larvicidal Activity of Artemisia vulgaris L. Stem Essential Oil against Aedes aegypti. 6(1): 11 - 16.

Habbachi, W., Benhissen, S., Ouakid, M.L., Farine, J.P et Bairi, A. 2014. Toxicity of aqueous extracts from Mediterranean plants on Culex pipiens (Mosquitoes). Case of Daphne gnidium (Thymelaeaceae) and Peganum harmala (Zygophyllaceae).Wulefenia journal, 21(12): 244-252.

Iauk, L., Mangano, K., Rapisarda, A., Ragusa, S., Maiolino, L., Musumeci, R., Costanzo, R., and Serra, A. 2004. J. Ethnopharm. 90: 267-272.

Kabouche, Z., Benkiki, N., Seguin, E., and Bruneau, C. 2003. A new dicoumarinyl ether and two rare furocoumarins from Ruta montana, Fitoterapia 74: 194-196.

Majdoub, O., Dhen, N., Souguir, S., Haouas, D., Baouandi, M., Laarif, A., and Chaieb, I. 2014. Chemical composition of Ruta chalepensis essential oils and their insecticidal activity against Tribolium castaneum.Tunisian Journal of Plant Protection 9: 83-90.

Meepagala, K.M., Schrader, K.K., Wedge, D.E., and Duke, S.O. 2005. Algicidal and antifungal compounds from the roots of Ruta graveolens and synthesis of their analogs. Phytochemistry 66: 2689-2695.

Merabti, B., Lebouz, I., Adamou, A., Ouakid, M.L. 2015. Effet toxique de 1'extrait aqueux des fruits de Citrullus colocynthis (L.) Schrad sur les larves des Culicidae. Revue des Bio Ressources, 5(2): 120-130.

Milesi, S., Massot, B., Gontier, E., Bourgaud, F., and Guckert, A. 2001. Ruta graveolens L.: a promising species for the production of furanocoumarins. Plant Science 161: 189-199.

Organisation Mondiale de la Santé.1963. Geneve 19-25 Novembre rapport annuel du directeur générale à l'assemblée mondiale de la santé et aux nations unies.

Raghav, S.K., Gupta B., Agrawal C., Goswami K., and Das H.R. 2006. Antiinflammatory effect of Ruta graveolens L. in murine macrophage cells. J. Ethnopharm. 104: 234- 239.

Tampe J., Parra L., Huaiquil K., Quiroz A., 2016. Potential repellent activity of the essential oil of Ruta chalepensis (Linnaeus) from Chile against Aegorhinus superciliosus (Guérin) (Coleoptera: Curculionidae). Journal of Soil Science and Plant Nutrition. 16 (1): 48-59.

Waterman, P.G. 1975. Alkaloids of the Rutaceae: their ditribution and Systematic Significance, Biochemical Systematics and Ecology. Ed. Pergamon Press, p: 149-180. 